\title{
Coulomb and surface effects on the pasta structure in nuclear matter
}

\section{Toshiki Maruyama*}

Japan Atomic Energy Research Institute, ${ }^{\dagger}$ Tokai, Ibaraki 319-1195, Japan

Email: maruyama.toshiki@jaea.go.jp

\section{Toshitaka Tatsumi}

Department of Physics, Kyoto University, Kyoto 606-8502, Japan

\section{Dmitri N. Voskresensky}

Moscow Institute for Physics and Engineering, Kashirskoe sh. 31, Moscow 115409, Russia

Gesellschaft für Schwerionenforschung mbH, Planckstr. 1, 64291 Darmstadt, Germany

\section{Tomonori Tanigawa}

Japan Society for the Promotion of Science, Tokyo 102-8471, Japan

Japan Atomic Energy Research Institute, ${ }^{\dagger}$ Tokai, Ibaraki 319-1195, Japan

\section{Tomoki Endo}

Department of Physics, Kyoto University, Kyoto 606-8502, Japan

\section{Satoshi Chiba}

Japan Atomic Energy Research Institute ${ }^{\dagger}$ Tokai, Ibaraki 319-1195, Japan

\begin{abstract}
Non-uniform structures in nuclear matter are studied by means of the density functional theory with relativistic mean fields. Our results demonstrate a particular role of the charge screening and the surface tension on the non-uniform structures.
\end{abstract}

29th Johns Hopkins Workshop on Current problems in Particle Theory: Strong Matter in the Heavens 1-3 August

Budapest

\footnotetext{
*Speaker.

†Present name: Japan Atomic Energy Agency
} 


\section{Introduction}

There have been several first-order phase transitions expected in the stellar objects: liquid-gas transition in supernova matter, neutron-drip, meson condensation and hadron-quark transitions in neutron stars. In such first-order transitions with more than one chemical potential, non-uniform mixed phases called "pasta" structures have been discussed as a consequence of phase equilibrium given by the Gibbs conditions. As density is increased, the geometrical structure of the mixed phase changes from droplet, rod, slab, tube, and to bubble. Naively speaking, pasta structures are expected to be realized due to the balance of surface tension and the Coulomb repulsion, which are called the finite-size effects. In the previous studies so far, however, the Coulomb interaction was treated rather simply and the surface tension was often introduced by hand.

We numerically study the non-uniform mixed phase by invoking the density functional theory with relativistic mean fields which includes the Coulomb interaction fully consistent with the charged particle distribution. The surface tension is naturally given by the spatial variation of the meson mean fields. With this framework, we discuss the effects of the Coulomb interaction and the surface tension on the pasta structure in the case of low density matter and kaonic pasta following $K^{-}$condensation at high densities. We clearly see an important effect, the charge screening effect, in our numerical results.

\section{Framework}

Following the idea of the density functional theory within the RMF model, we can derive the equations of motion to study non-uniform nuclear matter numerically, cf. [1]. In our framework, the Coulomb interaction is properly included in the equations of motion for nucleons, electrons, kaons and meson mean fields. Then we solve the Poisson equation for the Coulomb potential $V_{\text {Coul }}$ self-consistently with other equations. We start with the thermodynamic potential for the system of neutrons, protons, electrons, kaons $\left(K^{-}\right)$and meson mean fields,

$$
\Omega=\Omega_{N}+\Omega_{M}+\Omega_{e}+\Omega_{K}
$$

The first term

$$
\Omega_{N}=\sum_{a=p, n} \int d^{3} r\left[\int_{0}^{k_{\mathrm{F}, a}} \frac{d^{3} k}{4 \pi^{3}} \sqrt{m_{N}^{* 2}+k^{2}}-\rho_{a} v_{a}\right]
$$

is the contribution of nucleons with the local Fermi momenta $k_{\mathrm{F}, a}(\mathbf{r}) ; a=n, p$ and the effective nucleon mass $m_{N}^{*}(\mathbf{r})=m_{N}-g_{\sigma N} \sigma(\mathbf{r})$ with $m_{N}$ being the bare nucleon mass. Nucleons couple with the scalar $(\sigma)$, vector $\left(\omega\right.$ and $\rho$ ) mean fields to give $v_{n}(\mathbf{r})=\mu_{n}-g_{\omega N} \omega_{0}(\mathbf{r})+g_{\rho N} R_{0}(\mathbf{r})$, $v_{p}(\mathbf{r})=\mu_{p}+V_{\text {Coul }}(\mathbf{r})-g_{\omega N} \omega_{0}(\mathbf{r})-g_{\rho N} R_{0}(\mathbf{r})$, where $\mu_{n}$ and $\mu_{p}$ are neutron and proton chemical potentials and $g_{\sigma N}, g_{\omega N}$ and $g_{\rho N}$ are the coupling constants. The second term in (2.1) incorporates the contribution by the scalar and vector mean fields,

$$
\Omega_{M}=\int d^{3} r\left[\frac{(\nabla \sigma)^{2}+m_{\sigma}^{2} \sigma^{2}}{2}+U(\sigma)-\frac{\left(\nabla \omega_{0}\right)^{2}+m_{\omega}^{2} \omega_{0}^{2}}{2}-\frac{\left(\nabla R_{0}\right)^{2}+m_{\rho}^{2} R_{0}^{2}}{2}\right]
$$

where $m_{\sigma}, m_{\omega}$ and $m_{\rho}$ are the meson masses, and $U(\sigma)=\frac{1}{3} b m_{N}\left(g_{\sigma N} \sigma\right)^{3}+\frac{1}{4} c\left(g_{\sigma N} \sigma\right)^{4}$ is the nonlinear potential for the scalar field. The third term in (2.1) summarizes the contribution of the 
Coulomb potential $V_{\text {Coul }}(\mathbf{r})$ and the contribution of relativistic electrons,

$$
\Omega_{e}=\int d^{3} r\left[-\frac{1}{8 \pi e^{2}}\left(\nabla V_{\text {Coul }}\right)^{2}-\frac{\left(\mu_{e}-V_{\text {Coul }}\right)^{4}}{12 \pi^{2}}\right],
$$

where $\mu_{e}$ is the electron chemical potential. The last term in (2.1) is the thermodynamic potential of the kaon field $K=f_{K} \theta / \sqrt{2}$ with the kaon decay constant $f_{K} \simeq 93 \mathrm{MeV}$,

$$
\Omega_{K}=\int d^{3} r\left\{-\frac{f_{K}^{2} \theta^{2}}{2}\left[-m_{K}^{* 2}+\left(\mu_{K}-V_{\text {Coul }}+g_{\omega K} \omega_{0}+g_{\rho K} R_{0}\right)^{2}\right]+\frac{f_{K}^{2}(\nabla \theta)^{2}}{2}\right\},
$$

where $m_{K}^{*}(\mathbf{r})=m_{K}-g_{\sigma K} \sigma(\mathbf{r})$ is the effective $K^{-}$mass with $m_{K}$ being the bare kaon mass, $g_{\sigma K}$, $g_{\omega K}, g_{\rho K}$ are the coupling constants, and $\mu_{K}$ is the kaon chemical potential. The kaon charge density $\rho_{K}$ is expressed in terms of the kaon field $\theta$ as

$$
\rho_{K}=-\left(\mu_{K}-V_{\text {Coul }}+g_{\omega K} \omega_{0}+g_{\rho K} R_{0}\right) f_{K}^{2} \theta^{2} .
$$

Temperature $T$ is kept zero in the present study.

The equations of motion for the mean fields, kaon and the Coulomb potential are given by the variational principle $\frac{\delta \Omega}{\delta \phi_{i}(\mathbf{r})}=0\left(\phi_{i}=\sigma, \omega_{0}, R_{0}, \theta, V_{\text {Coul }}\right)$. Similarly the density functions for nucleons and electrons are given by $\frac{\delta \Omega}{\delta \rho_{a}(\mathbf{r})}=0(a=n, p, e)$. These equations read

$$
\begin{aligned}
\nabla^{2} \sigma(\mathbf{r})= & m_{\sigma}^{2} \sigma(\mathbf{r})+\frac{d U}{d \sigma}-g_{\sigma N}\left(\rho_{n}^{(s)}(\mathbf{r})+\rho_{p}^{(s)}(\mathbf{r})\right)+2 g_{\sigma K} m_{K} f_{K}^{2} \theta^{2}(\mathbf{r}), \\
\nabla^{2} \omega_{0}(\mathbf{r})= & m_{\omega}^{2} \omega_{0}(\mathbf{r})-g_{\omega N}\left(\rho_{p}(\mathbf{r})+\rho_{n}(\mathbf{r})\right) \\
& \quad-f_{K}^{2} g_{\omega K} \theta^{2}(\mathbf{r})\left(\mu_{K}-V_{\mathrm{Coul}}(\mathbf{r})+g_{\omega K} \omega_{0}(\mathbf{r})+g_{\rho K} R_{0}(\mathbf{r})\right), \\
\nabla^{2} R_{0}(\mathbf{r})= & m_{\rho}^{2} R_{0}(\mathbf{r})-g_{\rho N}\left(\rho_{p}(\mathbf{r})-\rho_{n}(\mathbf{r})\right) \\
& \quad-f_{K}^{2} g_{\rho K} \theta^{2}(\mathbf{r})\left(\mu_{K}-V_{\mathrm{Coul}}(\mathbf{r})+g_{\omega K} \omega_{0}(\mathbf{r})+g_{\rho K} R_{0}(\mathbf{r})\right), \\
\nabla^{2} \theta(\mathbf{r})= & {\left[m_{K}^{*}(\mathbf{r})^{2}-\left(\mu_{K}-V_{\mathrm{Coul}}(\mathbf{r})+g_{\omega K} \omega_{0}(\mathbf{r})+g_{\rho K} R_{0}(\mathbf{r})\right)^{2}\right] \theta(\mathbf{r}), } \\
\nabla^{2} V_{\mathrm{Coul}}(\mathbf{r})= & 4 \pi e^{2} \rho_{\mathrm{ch}}(\mathbf{r}), \quad\left(\operatorname{charge} \operatorname{density~} \rho_{\mathrm{ch}}(\mathbf{r})=\rho_{p}(\mathbf{r})+\rho_{e}(\mathbf{r})+\rho_{K}(\mathbf{r})\right), \\
\mu_{B}= & \mu_{n}=\sqrt{k_{F n}(\mathbf{r})^{2}+m_{N}^{*}(\mathbf{r})^{2}}+g_{\omega N} \omega_{0}(\mathbf{r})-g_{\rho N} R_{0}(\mathbf{r}), \\
\mu_{B}-\mu_{e}= & \mu_{p}=\sqrt{k_{F p}(\mathbf{r})^{2}+m_{N}^{*}(\mathbf{r})^{2}}+g_{\omega N} \omega_{0}(\mathbf{r})+g_{\rho N} R_{0}(\mathbf{r})-V_{\mathrm{Coul}}(\mathbf{r}) .
\end{aligned}
$$

The last two equations are the standard relations between the local nucleon densities and chemical potentials within the Thomas-Fermi approximation. We assume that the system is in $\beta$ equilibrium. Then we have only two independent chemical potentials, the baryon-number chemical potential $\mu_{B}=\mu_{n}$ and the charge chemical potential, i.e. the electron chemical potential, $\mu_{e}$, according to the corresponding conserved charges. Under the same assumption $\mu_{K}=\mu_{e}$.

To solve the above coupled equations numerically, the whole space is divided into equivalent Wigner-Seitz cells. The geometrical shape of the cell changes as follows: sphere in threedimensional (3D) calculation, cylinder in 2D and slab in 1D, respectively. Each cell is globally charge-neutral and all physical quantities in the cell are smoothly connected to those of the next cell with zero gradients at the boundary. Every point inside the cell is represented by the grid 

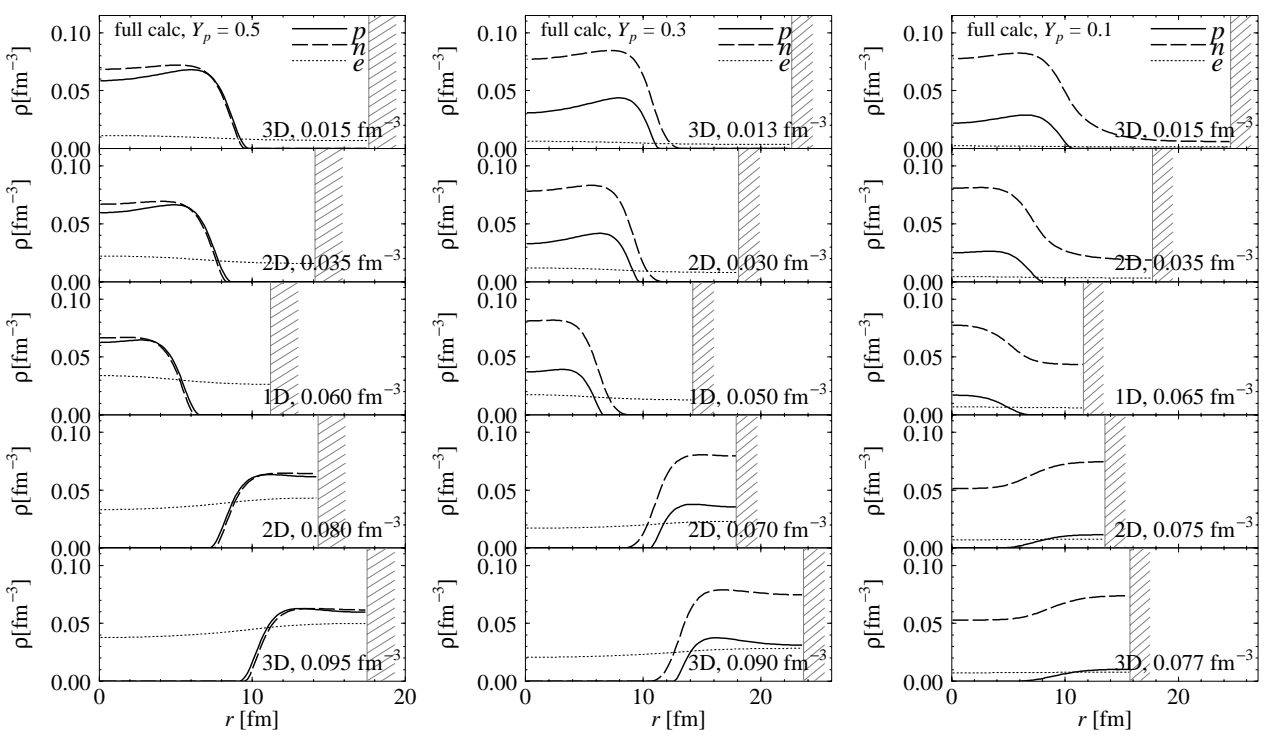

Figure 1: Examples of the density profiles in the cell for symmetric nuclear matter with $Y_{p}=0.5$ (left panel) and for asymmetric matter with $Y_{p}=0.3$ (center panel) and 0.1 (right panel).

points (number of grid points $N_{\text {grid }} \approx 100$ ) and differential equations for fields are solved by the relaxation method for a given averaged baryon-number density under constraints of the global charge neutrality. Details of the numerical procedure are explained in Ref. [2].

Parameters in the RMF model are chosen to reproduce saturation properties of nuclear matter: the minimum energy per nucleon $-16.3 \mathrm{MeV}$ at $\rho=\rho_{0} \equiv 0.153 \mathrm{fm}^{-3}$, the incompressibility $K\left(\rho_{0}\right)=240 \mathrm{MeV}$, the effective nucleon mass $m_{N}^{*}\left(\rho_{0}\right)=0.78 m_{N} ; m_{N}=938 \mathrm{MeV}$, and the isospinasymmetry coefficient $a_{\text {sym }}=32.5 \mathrm{MeV}$. Coupling constants and meson masses used in our calculation are as follows: $g_{\sigma N}=6.3935, g_{\omega N}=8.7207, g_{\rho N}=4.2696, b=0.008659, c=0.002421$, $m_{\sigma}=400 \mathrm{MeV}, m_{\omega}=783 \mathrm{MeV}, m_{\rho}=769 \mathrm{MeV}, m_{K}=494 \mathrm{MeV}, g_{\omega K}=g_{\omega N} / 3, g_{\rho K}=g_{\rho N}$, $U_{K}\left(\rho_{0}\right)=-130 \mathrm{MeV}$. We have checked that with these parameters and by including the Coulomb interaction, the binding energies of finite nuclei and the proton fraction, as well as the nucleon density profiles are well reproduced, except for very light nuclei [2].

\section{Pasta structures in nuclear matter}

First, we are concentrated on the discussion of the behavior of low-density nucleon matter at a fixed value of the proton number ratio $Y_{p}$. The cases $Y_{p}=0.3-0.5$ should be relevant for the supernova matter and for newly born neutron stars. In this fixed $Y_{p}$ case, a part of the condition (2.13), i.e. beta equilibrium, is not fulfilled. Note that at this low density case kaon does not appear: $\theta$ in Eq. (2.10) remains zero. Figure 1 shows some typical density profiles inside the Wigner-Seitz cells. The geometrical dimension of the cell is denoted as "3D" etc. The horizontal axis in each panel denotes the radial distance from the center of the cell. The cell boundary is indicated by the hatch. From the top to the bottom the configuration corresponds to droplet (3D), rod (2D), slab (1D), tube (2D), and bubble (3D). The nuclear "pasta" structures are clearly manifested. For the lowest $Y_{p}$ case $\left(Y_{p}=0.1\right)$, the neutron density is finite at any point: the space is filled by dripped 

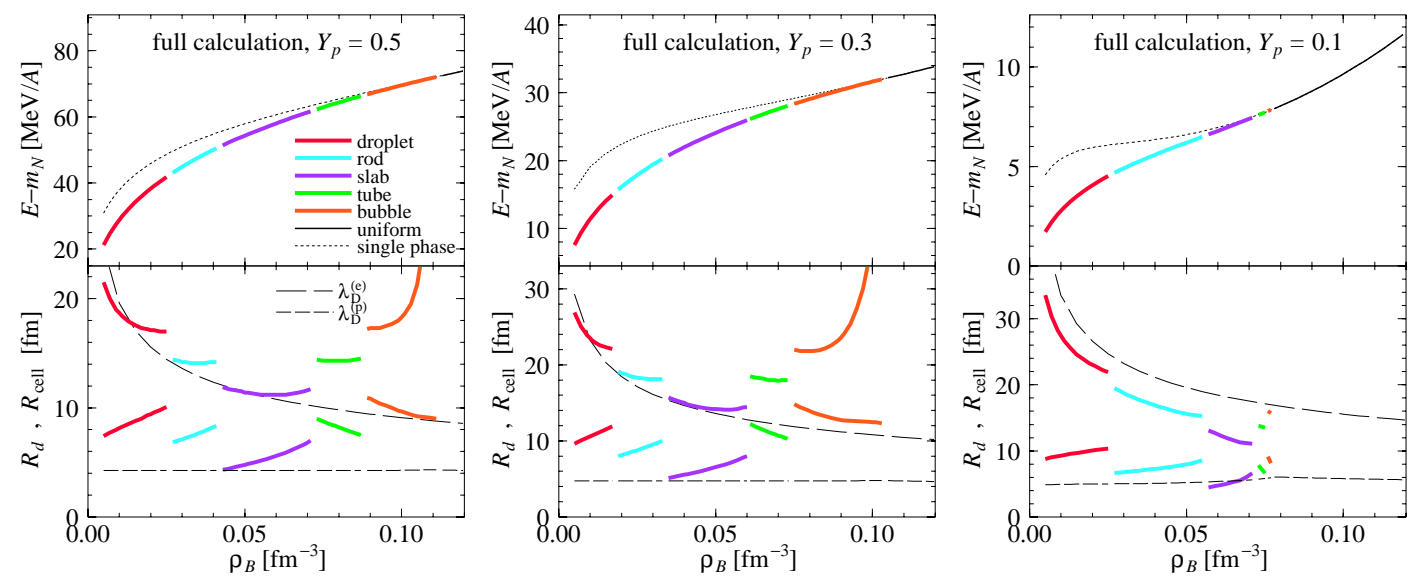

Figure 2: Binding energy per nucleon, and the cell and structure sizes, $R_{\text {cell }}$ and $R_{d}$, for nuclear matter with $Y_{p}=0.5,0.3$ and 0.1 .

neutrons. We can apparently see the charge screening effects: due to the spatial rearrangement of electrons the electron density profile becomes no more uniform. This non-uniformity of the electron distribution is more pronounced for a higher $Y_{p}$ and a higher density. Since protons repel each other, the proton density profile substantially deviates from the step-function: the proton density is enhanced near the surface of the nucleus.

The equation of state (EOS) for the sequence of geometric structures is shown in Fig. 2 (top panels) as a function of the averaged baryon-number density. Note that the energy $E-m_{N}$ also includes the kinetic energy of electrons, which makes the total pressure positive. The lowest-energy configurations are selected among various geometrical structures. The most favorable configuration changes from the droplet to rod, slab, tube, bubble, and to the uniform one (the dotted thin curve) with increase of density. The appearance of non-uniform structures in matter results in a softening of EOS: the energy per baryon gets lower up to about $15 \mathrm{MeV} / A$ compared to the uniform matter.

The bottom panels in Fig. 2 show the cell size $R_{\text {cell }}$ and the structure size $R_{d}$ (radii of droplet, rod, slab, tube and bubble) versus averaged baryon number density. Dashed curves show the Debye screening lengths of electron and proton, $\lambda_{D}^{(e)}$ and $\lambda_{D}^{(p)}$ for comparison. Numerically, the cell sizes $R_{\text {cell }}$ for droplet, rod, and slab configurations at $Y_{p}=0.5$ and 0.3 were proven to be close to the electron screening lengths. For the tube, $R_{\text {cell }}$ is larger than $\lambda_{D}^{(e)}$. For $Y_{p}=0.1$, in all cases $R_{\text {cell }}$ is substantially smaller than $\lambda_{D}^{(e)}$ and thereby the electron screening should be much weaker. In all cases, except for bubbles (at $Y_{p}=0.5$ and 0.3), the structure sizes $R_{d}$ are smaller than $\lambda_{D}^{(e)}$. This means that the Debye screening effect of electrons inside these structures should not be pronounced. For bubbles at $Y_{p}=0.5$ and $0.3, \lambda_{D}^{(e)}$ is substantially smaller than the cell size and the electron screening should be significant. For $Y_{p}=0.5,0.3,0.1$ in all cases (with the only exception $Y_{p}=0.1$ for slabs), the value $\lambda_{D}^{(p)}$ is shorter than $R_{d}$. Hence the density rearrangement of protons is essential for the pasta structures, as it is indeed seen from the Fig. 1.

We have also studied the structure of nucleon matter in beta equilibrium. We have found that only one type of structures is realized: proton-enriched droplets embedded in the neutron sea. No other geometrical structures like rod, slab, etc. appeared. One can note the similarity of the case of 


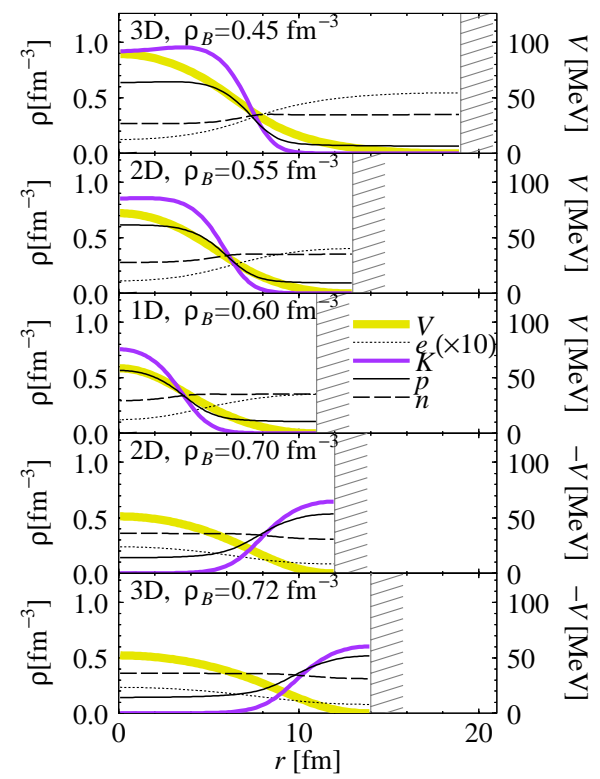

Figure 3: Density profiles of kaonic structures. Here the density does not mean charge-density but number-density of particles. Displayed using the right axis is the Coulomb potential $V_{\text {Coul }}$ (written as 'V').

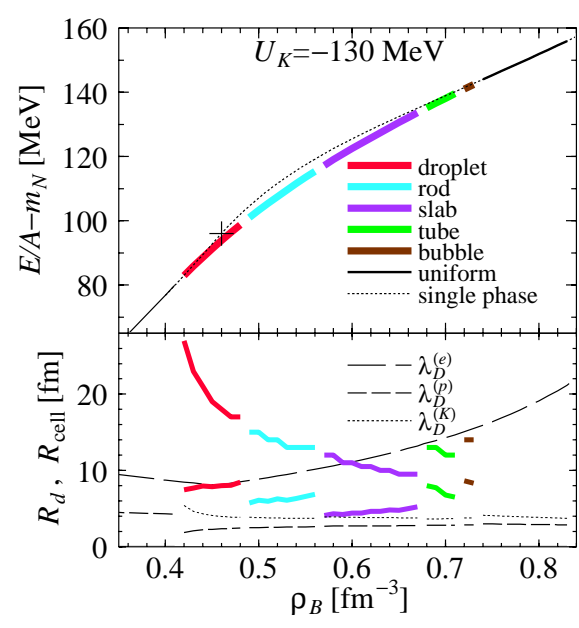

Figure 4: Top: Binding energy per nucleon of the nuclear matter in the beta equilibrium. The dotted line below the cross shows the uniform normal nuclear matter and above the cross, the uniform kaonic matter. Bottom: Structure size $R_{d}$ (thick curves below) and cell size $R_{\text {cell }}$ (thick curves above). Compared are the Debye screening lengths of electron, proton and kaon.

beta equilibrium and that of small proton ratio $\left(Y_{p}=0.1\right)$ where the droplet phase is more dominant.

Next we explore high-density nuclear matter in beta-equilibrium, which is expected in the inner core of neutron stars. Kaons are the lightest mesons with strangeness, and their effective energy is much reduced by the kaon-nucleon interaction in nuclear medium. For low-energy kaons the $s$ wave interaction is dominant and attractive in the $I=1$ channel, so that negatively charged kaons appear in the neutron-rich matter once the process $n \rightarrow p+K^{-}$becomes energetically allowed. Since kaons are bosons, it causes the Bose-Einstein condensation at zero momentum [3].

Since the kaon condensation is of the first order, it may give rise to non-uniform structure as a structured mixed phase. In fact, the system exhibits a series of structure change similar to the nuclear "pasta": the kaonic droplet, rod, slab, tube, bubble (which we call "kaonic pasta" structures). Figure 3 displays typical density profiles and the Coulomb potential. The neutron distribution proves to be rather flat. The proton distribution on the other hand is strongly correlated with the kaon distribution, which means that the Coulomb interaction is crucial.

In the upper panel of Fig. 4 we depict the energy per nucleon of the matter. The dotted line indicates the case of single phase (if one assumes absence of the mixed phase). In this case uniform matter consists of normal nuclear matter below the critical density and kaonic matter above the critical density. The cross on the dotted line $\left(\rho_{B} \simeq 0.46 \mathrm{fm}^{-3}\right)$ shows the critical density, i.e. the point where kaons begin to condensate in the case of single phase. Pieces of solid curves, on the other hand, indicate energetically favored structures. Droplets begin to appear for $\rho_{B}>0.41 \mathrm{fm}^{-3}$ smoothly decreasing the energy of the system. The mixed phase disappears for $\rho_{B}>0.74 \mathrm{fm}^{-3}$.

The lower panel of Fig. 4 shows the structure sizes $R_{d}$ and the cell sizes $R_{\text {cell }}$. We find that at the 

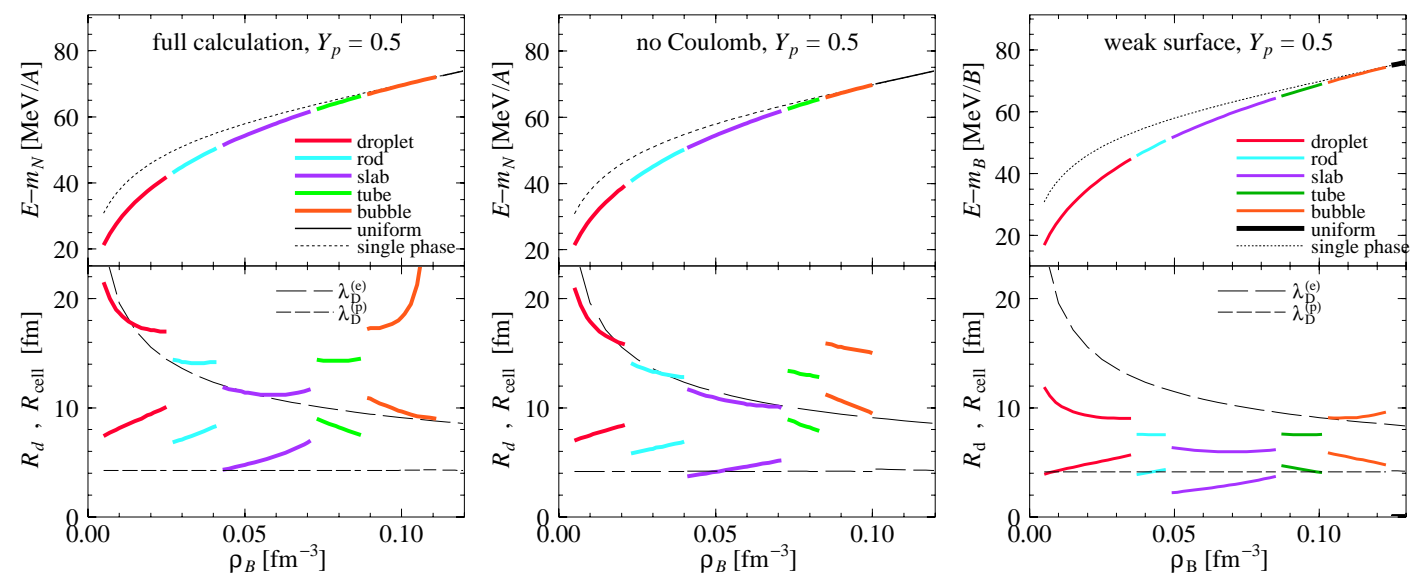

Figure 5: Comparison of the density profiles for different treatments of the Coulomb interaction. From the left: "full" calculation, "ho Coulomb" and "weak surface" calculation. The proton number ratio is $Y_{p}=0.5$ for all cases.
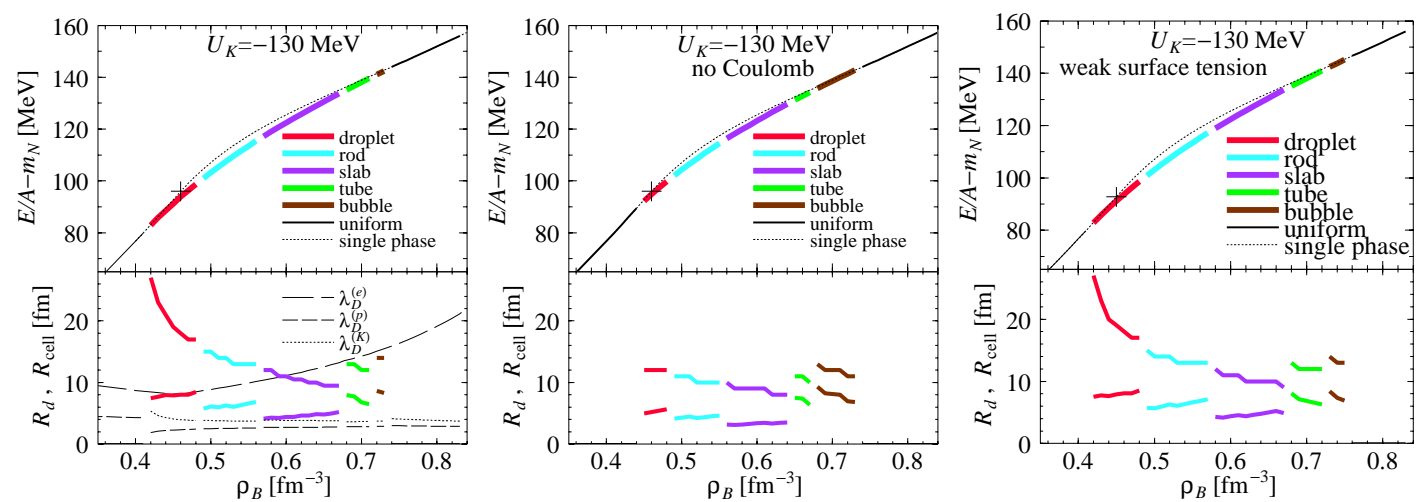

Figure 6: Top: the binding energy per nucleon of the nuclear matter in beta equilibrium. The electric potential is discarded determining the density profile and added evaluating the energy. Bottom: the structure size $R_{d}$ (thick curves below) and the cell size $R_{\text {cell }}$ (thick curves above).

onset density the size of the cell is infinitely large in case of the full calculation. The corresponding steep increase of $R_{\text {cell }}$ with decreasing density is clearly seen in the figure. The dashed lines and the dotted line in the lower panel of Fig. 4 show partial contributions to the Debye screening lengths of the electron, proton and kaon, $\lambda_{D}^{(e)}, \lambda_{D}^{(p)}$, and $\lambda_{D}^{(K)}$, respectively. We see that in most cases $\lambda_{D}^{(e)}$ is less than the cell size $R_{\text {cell }}$ but it is larger than the structure size $R_{d}$. The proton Debye length $\lambda_{D}^{(p)}$ and the kaon Debye length $\lambda_{D}^{(K)}$, on the other hand, are always shorter than $R_{\text {cell }}$ and $R_{d}$.

\section{Charge screening effect}

To demonstrate the charge screening effects we compare results of the full calculation with those given by a "perturbative" treatment of the Coulomb interaction often used in the literature, "no Coulomb" calculation. The electric potential is discarded in equations of motion (2.6)-(2.10), (2.12), (2.13) which determine the density profiles. The Coulomb energy is then added to the total 


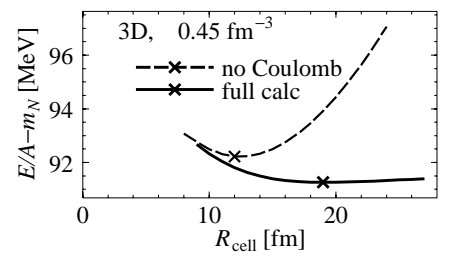

Figure 7: The cell size $R_{\text {cell }}$ dependence of the energy per nucleon. Crosses indicate the minimum points.

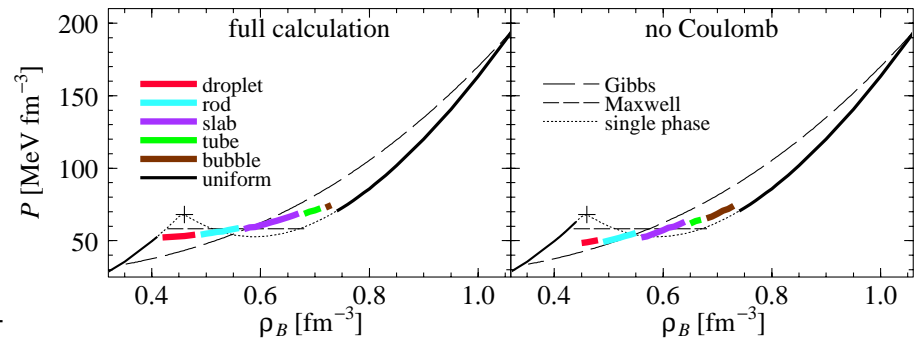

Figure 8: Pressure versus the baryon number density.

energy by using the charge density profile thus determined to find the optimal value with respect to the cell size $R_{\text {cell }}$.

First, we discuss the case of low-density symmetric nuclear matter. In the left and the central panels of Fig. 5, compared are different treatments of the Coulomb interaction. The EOS (upper panels) as a whole shows almost no dependence on the treatments of the Coulomb interaction. However, sizes of the cell and the nucleus (lower panels), especially for tube and bubbles, are different. In the cases of the "full calculation", the cell radii of "tube" and "bubble" structures and that of "slab" structure get larger with increase of density, while they are monotonically decreasing in the case of "no Coulomb" calculation. The other effect is a difference in the density range for each pasta structure. The "full" treatment of the Coulomb interaction slightly increases the region of the nuclear pasta.

We show the same comparison for kaonic pasta structure in Fig. 6. We see again that the density range of the mixed phase is narrower in the case of the "no Coulomb" calculation than in the full calculation, while the energy gain is almost the same. A remarkable difference is seen in the cell size, especially near the onset density of kaonic pastas, for $\rho_{B}<0.5 \mathrm{fm}^{-3}$. The cell size given by the full calculation is always larger than that given by the "no Coulomb" calculation.

To elucidate the screening effect, we depict the $R_{\text {cell }}$ dependence of the energy per nucleon in Fig. 7. In a general case of 3D droplet the Coulomb energy per particle depends on the radius by its square, while the surface energy per particle by its inverse. Therefore the sum of the Coulomb and surface energy has a U-shape (cf. "no Coulomb") and has a minimum at a certain radius. If the Coulomb interaction is screened, the Coulomb part will be suppressed (cf. the full calculation) and the minimum point gets larger. Since the cell radius is approximately proportional to the droplet radius for a given baryon density, the above argument applies also to the cell size.

For a long time there existed a naive view that not all the Gibbs conditions can be satisfied in a description by the Maxwell construction if there are two or more independent chemical components $[4,5,6,7]$, because the local charge neutrality is implicitly assumed in it. As the result of this argument, it was suggested that a broad region of structured mixed phase may appear in neutron stars. However, in recent papers $[8,9,10,11,12]$ we have demonstrated that if one properly includes the Coulomb interaction, the Maxwell construction practically satisfies the Gibbs conditions and the range of the mixed phase will be limited.

We present in Fig. 8 the pressure as a function of the baryon-number density. We also depict the pressure when the Gibbs conditions are applied for two semi-infinite matters disregarding the Coulomb interaction (indicated by "Gibbs") and that given by the Maxwell construction (indicated 

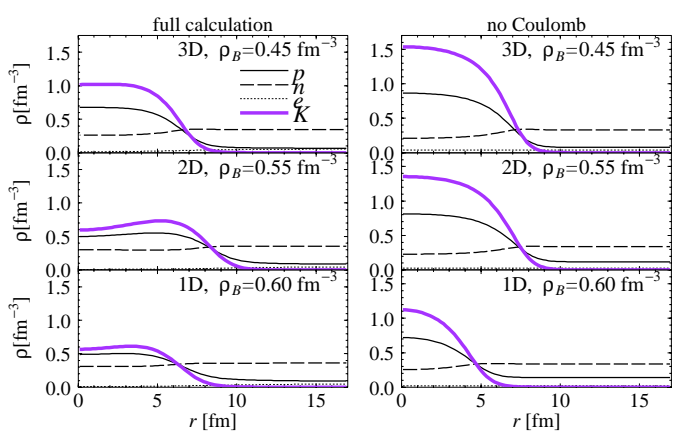

Figure 9: Comparison of density profiles of kaonic matter in full and "ho Coulomb" calculations. The cell size, $R$ cell $=$ $17 \mathrm{fm}$, is not optimized since the optimum values would be different for different calculations.

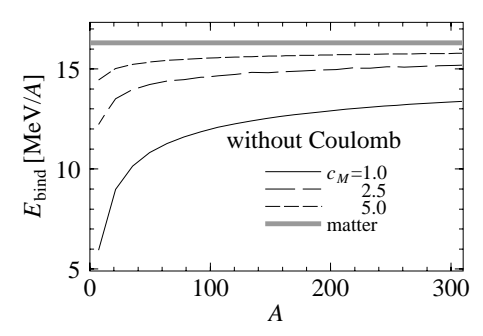

Figure 10: Mass number $A$ dependence of the binding energy of finite nuclei without the Coulomb interaction. Thick gray line shows the case of nuclear matter.

by "Maxwell"). We see that the pieces of solid curves lie between "Gibbs" and "Maxwell". The full calculation case is more similar to the one given by the Maxwell construction. In Fig. 9 compared are the density profiles obtained by the full and "no Coulomb" calculations. In case of the full calculation the difference between the negative charge density (of kaons and electrons) and the positive charge density of protons is smaller, indicating that the system tends to have a local charge neutrality. These results suggest that the Maxwell construction is effectively justified in the full calculation owing to the charge screening effects.

\section{Surface effects}

If we artificially multiply meson masses $m_{\sigma}, m_{\omega}$ and $m_{\rho}$ by a factor $c_{M}$, e.g. $c_{M}=1$ (realistic case), 2.5 and 5.0, the surface tension changes. Figure 10 demonstrates the binding energies of finite nuclei calculated with different meson masses. By the use of heavy meson masses, the binding energy of finite nuclei (for finite $A$ ) approaches to that of nuclear matter indicated by a thick gray line. This shows that the surface tension is reduced with increase of the meson masses, cf. [13]. Notice that this statement is correct only if we fix the ratio $g_{\phi N}^{2} / m_{\phi}^{2}$.

Using the above modified meson masses, we explore the effects of surface tension in the following. Right panels of Figs. 5 and 6 are EOS and the cell and structure sizes, but now for the case of an artificially suppressed surface tension $\left(c_{M}=5.0\right)$. Comparing them with the left panels, we see that there is almost no difference in the EOS. However, there are two differences in the case of low-density nuclear matter. First, the density range of pasta structure is slightly broader for weaker surface tension. Secondly, the cell size with a normal surface tension is larger than the case of weaker one. It means that weaker surface tension and stronger Coulomb repulsion cause the similar effects on the cell size since the pasta structure is realized by the balance of the both.

In the case of kaonic pasta, the meson masses have very small effects. The $\sigma, \omega$ and $\rho$ mesons have less contribution to the surface tension of kaonic pasta but $K^{-}-N$ interaction is dominant.

\section{Summary}

We have discussed two kinds of non-uniform structures in nuclear matter, nuclear pasta at 
subnuclear density and the kaon mixed phase at high density, which may arise as consequences of the first order phase transitions with many particle species. Based on density functional theory and relativistic mean-field theory, we took into account the Coulomb interaction in a proper way and numerically solved coupled equations of motion to extract the density profiles.

In nuclear matter with fixed proton fractions, which is relevant for the supernova explosions and the newly born neutron stars, we have observed "nuclear pasta" structures. The appearance of the pasta structures significantly lowers the energy, i.e. softens the equation of state, while the energy differences between various geometrical structures are rather small.

By comparing different treatments of the Coulomb interaction, we have seen that the selfconsistent inclusion of the Coulomb interaction changes the phase diagram and the size of pasta structures. In particular the region of pasta structure is broader for "full" calculation compared to that with simplified treatments of the Coulomb interaction used in the previous studies. In the full calculation, the Coulomb screening (rearrangement of the charge distribution) lowers the energy and consequently enlarges the structure. We have also investigated the effects of surface tension caused by the gradient terms of $\sigma, \omega$ and $\rho$ mesons. By changing the contribution of these gradient terms, we have found that weaker surface tension and stronger Coulomb repulsion have the similar effects on the pasta structure.

Around the critical density of kaon condensation we have observed "kaonic pasta" similar to "nuclear pasta". The effects of the charge screening was found to be similar to those for nuclear pasta at low densities. Besides, the system tends to be local charge neutral and the Maxwell construction is effectively valid for the description of the mixed phases. The effects of $\sigma, \omega$ and $\rho$ mesons for the surface tension, however, was found to be small. Instead, the attraction between kaon and nucleon is dominant for the surface tension.

\section{References}

[1] R. G. Parr and W. Yang, Density-Functional Theory of Atoms and Molecules (Oxford U Press, 1989) Density Functional Theory, ed. E. K. U. Gross and R. M. Dreizler, Plenum Press (1995).

[2] T. Maruyama et al., nucl-th/0503027; Phys. Rev. C72, 015802 (2005).

[3] D. B. Kaplan and A. E. Nelson, Phys. Lett. B175, 57 (1986); B179, 409(E) (1986).

[4] N. K. Glendenning and J. Schaffner-Bielich, Phys. Rev. C 60, 025803 (1999).

[5] M. Christiansen and N. K. Glendenning, astro-ph/0008207.

[6] M. Christiansen, N. K. Glendenning and J. Schaffner-Bielich, Phys. Rev. C62, 025804 (2000).

[7] N. K. Glendenning, Phys. Rev. D46, 1274 (1992); Phys. Rep. 342, 393 (2001).

[8] D. N. Voskresensky, M. Yasuhira and T. Tatsumi, Phys. Lett. B541, 93 (2002);

T. Tatsumi and D. N. Voskresensky, nucl-th/0312114.

[9] D. N. Voskresensky, M. Yasuhira and T. Tatsumi, Nucl. Phys. A723, 291 (2003).

[10] T. Tatsumi et al., nucl-th/0502040.

[11] T. Endo, T. Maruyama, S. Chiba and T. Tatsumi, nucl-th/0410102; hep-ph/0502216.

[12] T. Maruyama et al., Nucl. Phys. A749, 186 (2005).

[13] W. D. Myers and W. J. Swiatecki, Nucl. Phys. A601, 141 (1996). 